Research Article

\title{
Probabilistic Analysis of Wheel Loader Failure under Rockfall Conditions Based on Bayesian Network
}

\author{
Zhenmin Feng $\left(\mathbb{D}\right.$, Dongmei Huang $\left(\mathbb{D}\right.$, Zhian Li ${ }^{(D)}$, Rui Li ${ }^{(D)}$, and Yupeng Sun $(\mathbb{D}$ \\ College of Quality and Safety Engineering, China Jiliang University, Hangzhou, Zhejiang 310018, China \\ Correspondence should be addressed to Dongmei Huang; dmhuang@cjlu.edu.cn and Zhian Li; lizhian_hz@163.com
}

Received 13 April 2021; Revised 14 July 2021; Accepted 13 October 2021; Published 31 October 2021

Academic Editor: Kauko Leiviskä

Copyright (c) 2021 Zhenmin Feng et al. This is an open access article distributed under the Creative Commons Attribution License, which permits unrestricted use, distribution, and reproduction in any medium, provided the original work is properly cited.

Rockfall is one of the most serious geological hazards in mountain regions. During the rescue situations after rockfall, the wheel loader, a vital type of modern engineering mechanism, plays an important role in relieving the obstruction of the catastrophic site. Increasing the reliability of the wheel loader during the rescue situation is quite important. This study aims to build a fault diagnosis model based on Bayesian network (BN) to diagnose the probability and path of the fault occurrence in the wheel loader during a rockfall disaster. Meanwhile, to reduce the influence of subjective factors, the fuzzy set theory is introduced into BN. The result showed that the probability of failure of the wheel loader under rockfall disaster is $13.11 \%$. In addition, the key cause of the failure of the wheel loader under the rockfall disaster is the malfunction of mechanical parts. The probability of mechanical component failures in this case is as high as $88 \%$, while the probability of human error is $6 \%$. The research results not only show the ability of the $\mathrm{BN}$ to incorporate subjective judgment but also can provide a reference for fault diagnosis and risk assessment of wheel loaders under rockfall disaster conditions.

\section{Introduction}

Rockfall is one of the most serious geological disasters in the mountain regions. Rockfall disaster refers to the phenomenon of irregular loose rocks falling to the foot of hill slopes or low-lying areas along the vertical or subvertical cliff under the gravity condition, which can lead to severe damage to infrastructure, personnel, and so on $[1,2]$. During recent decades, many rockfall events have happened in China. For example, 46 rockfalls occurred in 20 years between Dadan and Junshi Temple of Baocheng Railway, which damaged the tracks and vehicles and caused many parking accidents [3]; the rock collapse in Kangdinggas Gully section, Sichuan Province, leads to the interruption of traffic in 2017 [4]; the rockfall in Diecai Mountain Scenic Area in Guilin occurred causing 7 deaths and 25 injuries in 2015; the mountain collapse in Sichuan Province occurred and hit a bus causing 8 deaths and 16 injuries in 2009.

Wheel loader, which can be used in handling rockfall, dredging, and clearing the collapse site, is an important rescue construction machinery at the geological disaster rescue site. During rescue conditions, the machinery is easily affected by the impact of falling rocks leading to loss of rescue capability or decrease in rescue efficiency [5]. The research on the failure probability of the wheel loader under the condition of rockfall disaster is helpful to determine the fault causes as soon as possible, reduce the rescue risk, and avoid unnecessary loss.

Therefore, many researchers pay attention to the wheel loader failure under different conditions. Hang et al. [6] analyzed the possibility of failure of a wheel loader based on the safety system engineering theory. Jun and Xutao [7, 8] identified the fault components of the loader by the control engineering theory. However, most of the past studies usually refer to the problems of the loader system itself under normal working environment, such as oil spill during loading and unloading or failure of continuous working cooling system. The influence of environmental conditions on the operation of the loader is usually ignored. The reality is that loaders often work in harsh environments, such as mudslides, rockfalls, landslides, and forest areas with slippery terrain, which make it difficult to find faults and 
diagnose faults in time [9]. In this context, it is urgent to study fault diagnosis methods for the loaders under specific disaster situations so as to provide a theoretical basis for the environmental stability of the loader in the future [10].

In the past ten years, $\mathrm{BN}$, an important probabilistic graphical model, has been deeply studied and widely used in many engineering fields such as reliability engineering [11-14], risk assessment [15], ecological environment field $[16,17]$, social management field [18], and engineering field [19]. Kabir et al. [20] applied the Bayesian network to the safety performance evaluation of the oil and gas pipeline and found out the most important fault causes of oil and gas pipeline leakages. Han et al. [21] used the Bayesian network to determine the probability of failure in a logistics UAV during normal operation and the main reason for falling was battery failure. Yazdi and Kabir [22] incorporated fuzzy set theory into the Bayesian network to evaluate the reliability of ethylene transportation line. The above studies show that, compared with traditional methods, Bayesian networks can not only represent the internal relations among the faults of a complex multisystem but also effectively diagnose the faults of the system quantitatively. Therefore, the Bayesian network can comprehensively and quantitatively analyze the fault of wheel loaders.

However, when the system reliability analysis is studied, due to the complexity of the research object, the Bayesian network model will be relatively large, and the reasoning and calculation will become difficult and consume more time. Subsequently, many types of application software appeared one after another, and the common software includes Bayesian network software, GeNIe, Netica, and MATLAB software (BNT) based on FullBNT1.07 toolbox. In recent years, GeNIe software with its visual window is easy to operate the advantages of easy to use, more and more applications, and solving Bayesian network model applications. Zhang and Thai [23] sought to fill the gap by exploring the extension of $\mathrm{BN}$ with interval probabilities to the modeling of maritime accidents, which allows for the quantification of epistemic uncertainty. GeNIe software is used to establish the Bayesian network topology and calculate the posterior probability of each node. Shuting [24] used MATLAB and GeNIe software to simulate the Bayesian network of the braking control subsystem in the braking system. After comparing the learning effect and accuracy of the two, it is found that the GeNIe visualization window has the advantages of convenient operation, fast learning speed, and high calculation accuracy, so it is more suitable for the reliability analysis of the Bayesian network of the braking system. Therefore, this paper uses GeNIe2.3 to construct a Bayesian network and calculate the probability of probabilistic inference part of the Bayesian network.

The present work aims to build a dynamic probabilistic analysis framework of wheel loader failures based on the Bayesian network to improve the stability of loaders in the rescue process of rockfall disaster. A fault diagnosis model by the Bayesian-based approach was established based on the fault characteristics of the wheel loader, and the quantification of the model was based on expert's judgments. At the same time, to reduce the influence of subjective factors, the fuzzy set theory was introduced into the Bayesian network, and the triangular fuzzy number was used to modify the prior probability. Then, GeNIe2.3, which is one of the most common and useful types of software to deal with graphical models, was used to calculate the probability of the wheel loader faults. Finally, through the analysis of the posterior probability, the influence degree of each causative factor on the fault occurrence was quantified, and the maximum causal chain of the wheel loader was obtained.

\section{BN Fault Diagnosis Model Based on Fuzzy Theory}

\subsection{The Basic Theory of $B N$}

2.1.1. The Definition of BN. Bayesian network (BN), also known as belief network or causal network, is a technology that combines probability analysis and graph theory to describe and infer uncertain knowledge [25]. BN consists of two parts: one is a directed acyclic graph (DAG), which uses Bayesian network topology to describe the causal relationship between variables; the other part is a conditional probability table (CPT), which is used to deal with the uncertainty caused by conditional correlation. Its mathematical expression is

$$
B N=((N, \operatorname{Arc}), P) .
$$

In equation (1), $(N, \mathrm{Arc})$ indicates $\mathrm{DAG}$ and $P$ indicates the conditional probability table, where $N=\left(N_{1}, N_{2}, \ldots, N_{n}\right)$ is any nodes, which represent various variables, and Arc is the set of all directed edges in DAG, where each directed edge represents the direct dependency between nodes.

Conditional independence is a characteristic of Bayesian networks. In the case of determining a specific parent node $\mathrm{Pa}\left(N_{i}\right)$, any node $N_{i}$ in Bayesian network is conditionally independent of all nondescendant nodes $S\left(N_{i}\right)$ of $N_{i}$ :

$$
p\left(N_{i} \mid P a\left(N_{i}\right), S\left(N_{i}\right)\right)=p\left(N_{i} \mid P a\left(N_{i}\right)\right) .
$$

According to the assumption of conditional independence, the joint probability distribution of $n$ variables in Bayesian networks can be factorized as follows:

$$
p\left(N_{1}, N_{2}, \ldots, N_{n}\right)=\prod_{i=1}^{n} p\left(N_{i} \mid \operatorname{Pa}\left(N_{i}\right)\right),
$$

where $N_{1}, N_{2}, \ldots, N_{n}$ represent a variety of arbitrary node variables, $\mathrm{Pa}\left(N_{i}\right)$ is the parent node of $N_{i}$, and $p\left(N_{i} \mid P a\left(N_{i}\right)\right)$ is the occurrence probability under the condition of $\mathrm{Pa}\left(N_{i}\right)$.

The core of Bayesian theory is based on the conditional probability derived from Bayesian formulas to analyze the research object. Bayes' formula is as follows:

$$
p(A \mid B)=\frac{p(A) p(B \mid A)}{p(B)},
$$


where $p(A)$ and $p(B)$ are prior probabilities, $p(A \mid B)$ is a posterior probability, and $p(B \mid A)$ is a likelihood probability.

2.1.2. The Inference of $B N$. Bayesian network not only can describe the polymorphism of events and the uncertainty of fault probability but also has the advantages of bidirectional reasoning including causal reasoning and diagnostic reasoning [26].

Causal reasoning (i.e., top-down reasoning) refers to the forward reasoning according to the joint probability distribution of a given root node for obtaining the occurrence probability of the leaf nodes. Let the root node be $X_{i}(i=1,2, \ldots, n)$, the intermediate node be $M_{j}(j=1,2, \ldots, m)$, and the leaf node be $T$ in a Bayesian network. The corresponding node states are represented by $X_{i}^{a_{i}}, M_{j}^{b_{j}}$, and $T_{q}$, respectively, where the number of fault states of a root node is $a_{i}=1,2, \ldots, s$. Then the calculation formula of the probability of the leaf node in $T_{q}$ state is as follows:

$$
p\left(T=T_{q}\right)=\sum p\left(X_{1}, \ldots, X_{n}, M_{1}, \ldots, M_{n}, T=T_{q}\right) .
$$

Diagnostic reasoning (i.e., bottom-up reasoning) is used to calculate the occurrence probability of each root node when the leaf node is in different states [27]:

$$
p\left(X_{i}=X_{i}^{a_{i}} \mid T=T_{q}\right)=\frac{p\left(X_{i}=X_{i}^{a_{i}}\right) p\left(T=T_{q} \mid X_{i}=X_{i}^{a_{i}}\right)}{p\left(T=T_{q}\right)},
$$

where $p\left(X_{i}=X_{i}^{a_{i}} \mid T=T_{q}\right)$ represents the conditional probability of the root node $X_{i}$ being in state $X_{i}^{a_{i}}$ when the node $T$ is in state $T_{q} \cdot p\left(X_{i}=X_{i}^{a_{i}}\right)$ is the probability of occurrence when the root node $X_{i}$ is in state $X_{i}^{a_{i}}$. $p\left(T=T_{q} \mid X_{i}=X_{i}^{a_{i}}\right)$ is the conditional probability of the leaf node $T$ being in state $T_{q}$ when node $X_{i}$ is in $X_{i}^{a_{i}}$ state, and $p\left(T=T_{q}\right)$ is the probability of occurrence when the leaf node $T$ is in state $T_{q}$.

2.2. BN Fault Diagnosis Modeling Process. The modeling of Bayesian network fault diagnosis is mainly divided into the following stages [11]:

(i) Determine the node variables and their corresponding states

(ii) Determine structure modeling of $\mathrm{BN}$ according to the dependency relationship among nodes

(iii) Determine parameter modeling of $\mathrm{BN}$ which consists of prior probability of root nodes and conditional probability of leaf nodes by using expert knowledge and experience, statistical results of historical simulated, or experimental data

(iv) Determine posterior probability by $\mathrm{BN}$ inference for system faults identification
Based on the basic principle of the Bayesian network, the procedures of establishing Bayesian network fault diagnosis modeling are shown in Figure 1.

2.3. The Application of Fuzzy Set Theory. In the process of Bayesian network analysis, the accurate prior probability value can be determined by a parameter learning algorithm for a simple system with sufficient sample data. However, in the field of equipment fault diagnosis, the difficulty of acquiring prior probability increases, which is due to the complexity of diagnostic objects, limitations of testing methods, imprecision of knowledge, and the majority of uncertainty problems. To solve the problem of insufficient statistical samples, the Delphi method of expert judgment and fuzzy set theory is used to determine the prior probability of each root node in the Bayesian network. Further details are as follows.

2.3.1. Obtaining Linguistic Variables of Unknown Nodes by Delphi Method. Fuzzy set theory is an objective attribute of things that exists in many events in life. For example, the occurrence of faults has obvious characteristics of uncertainty, which is difficult to express in accurate language. The occurrence probability of events can be defined by language variables in order to obtain expert judgment initially, which are divided into seven classes by introducing seven linguistic variables: "very low," "low," "medium-low," "medium," "medium-high," "high," and "very high" [28].

Delphi method [29], which was by Rand Corporation in the early 1950s, is one of the most widely used methods in expert judgment. Communication mode is adopted in this method to make a centralized judgment, which can well solve the difficulty of mathematical model description and the uncertainty of calculation. The specific way is that several experts were consulted for this purpose and were furnished with a questionnaire by e-mail to provide their judgment about the failure possibility of root nodes.

\subsubsection{Transforming Linguistic Variables into Corresponding} Fuzzy Numbers. In fuzzy set theory, there are many applications to convert linguistic variables into fuzzy numbers such as triangles [30], trapezoids [31], and Gauss fuzzy membership function [32]. Wickens [33] divides the occurrence probability of events into seven linguistic variables, and its fuzzy membership distribution is shown in Figure 2.

The criterion of the most practical membership function is based on realistic circumstances [34]. In earlier studies, the triangular fuzzy number, proposed by PJM van Laarhoven and W. Pedrycz, is among the most widely used for its simple operation in reliability assessment [35-38]. The reason for using the triangular fuzzy number is that, under some weak assumptions, the defined membership functions can directly meet the appropriate optimization criteria [39]. The triangular fuzzy number is $P=\left(P_{L}, P_{M}, P_{H}\right)$, and its membership function $\mu_{P}(x)$ is expressed as follows: 


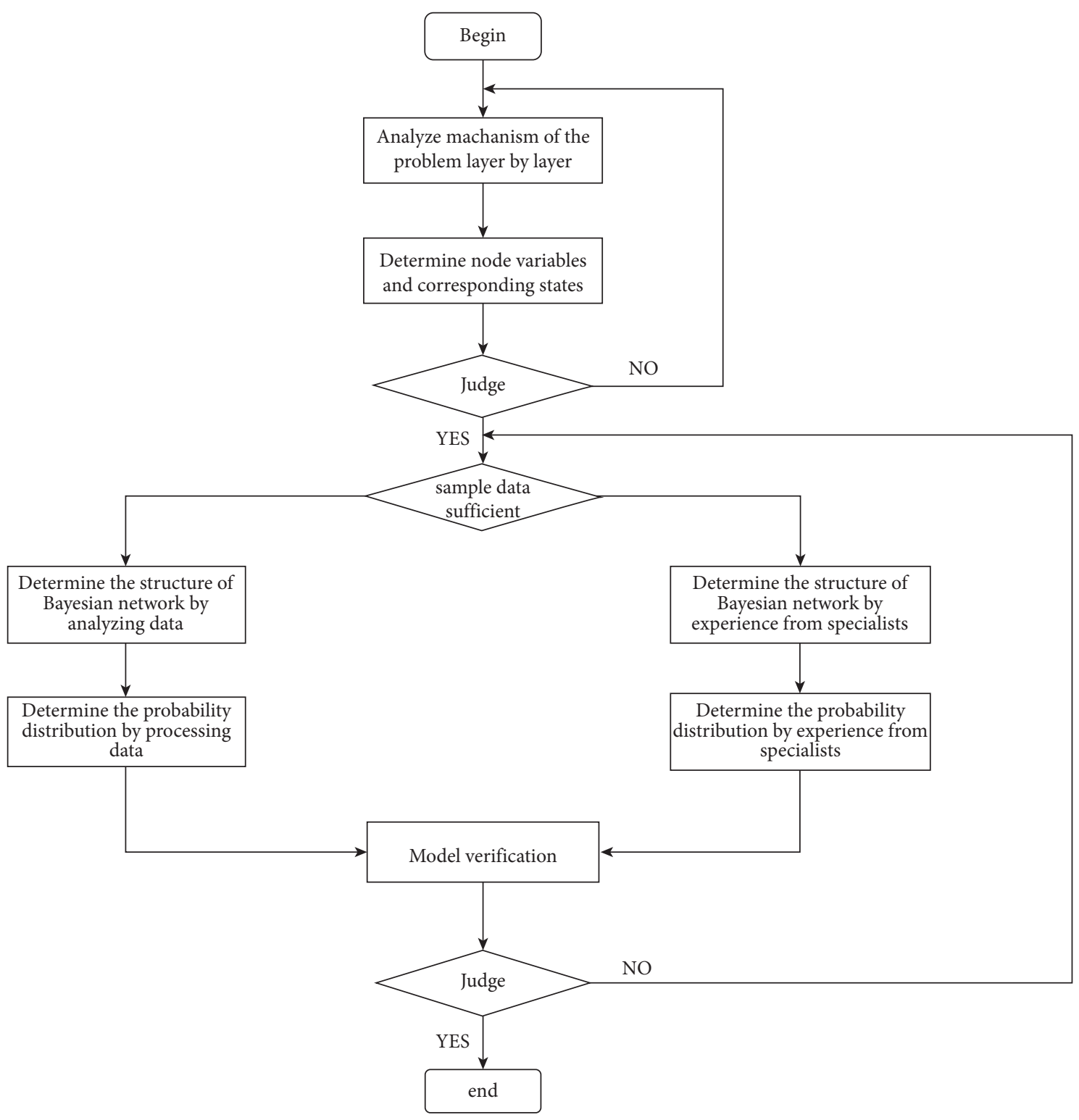

FIgURE 1: Fundamental procedure of Bayesian network.

$$
\mu_{P}(x)= \begin{cases}0, & \left(x<P_{L}\right), \\ \frac{x-P_{L}}{P_{M}-P_{L}}, & \left(P_{L} \leq x \leq P_{M}\right), \\ \frac{P_{H}-x}{P_{H}-P_{M}}, & \left(P_{M}<x \leq P_{H}\right), \\ 0, & \left(x>P_{H}\right) .\end{cases}
$$

In equation (7), $P_{L}$ and $P_{H}$ represent the upper and lower bounds of the event probability, respectively. The degree of fuzziness is determined by $P_{H}-P_{L}$, and the greater the difference, the greater the degree of fuzziness. $P_{M}$ is the mean value.
2.3.3. Transforming Fuzzy Numbers into Corresponding Prior Probability. Defuzzification refers to the process of taking a single value which can best represent the fuzzy set. To ensure that the prior probability is converted from a fuzzy value to an accurate value, the obtained expert evaluation results should be defuzzified before the Bayesian network inference calculation. In other words, the prior probability must be a crisp value in the inference calculation of Bayesian networks. Therefore, it is necessary to deal with the results of expert evaluation. If the probability of failure of each basic event in the Bayesian network is difficult to obtain or the fault data of new parts is less, it is completely dependent on the experience of experts to obtain the prior probability of each basic event. In the process of expert evaluation, according to their own experience, most experts use a triangular fuzzy number to replace the crisp value of each basic fault, so it is simple 


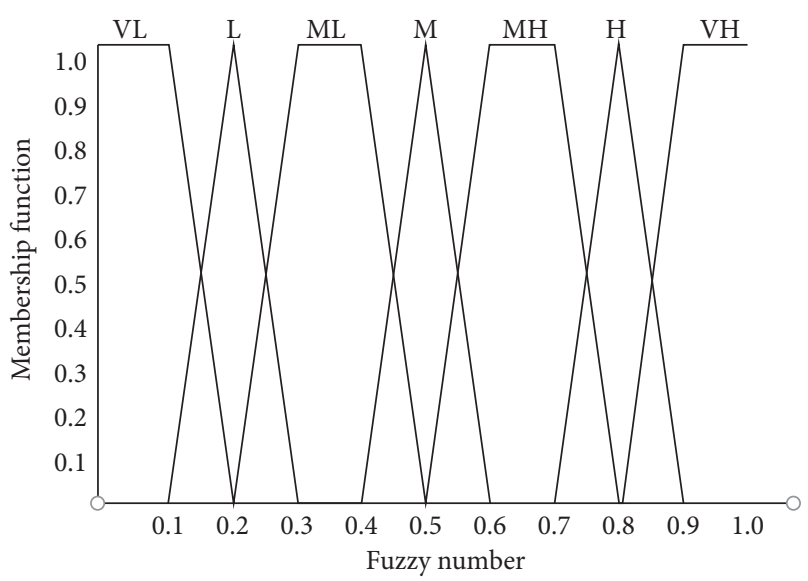

FIGURE 2: Fuzzy membership distribution of linguistic variables of Wickens.

and convenient to use mean area method to defuzzify [40]. Under such a background, more and more scholars choose this method to transform fuzzy failure probability into crisp failure probability [27]. Hence, the mean area [41, 42] is used to defuzzify the fuzzy failure rate to obtain the accurate fuzzy probability $E\left(\widetilde{P}_{i}\right)$ of the $i$ event in this paper. The calculation formula is as follows:

$$
E\left(\widetilde{P}_{i}\right)=\frac{P_{L i}+2 P_{M i}+P_{H i}}{4} .
$$

The accurate fuzzy probability $E\left(\widetilde{P}_{i}\right)$ is normalized to make it more consistent with the actual probability of the root node. The actual probability of the event is obtained from equations (9) and (10).

$$
\begin{aligned}
& P_{i}= \begin{cases}\frac{1}{10^{\alpha},} & E\left(\widetilde{P}_{i}\right) \neq 0, \\
0, & E\left(\widetilde{P}_{i}\right)=0,\end{cases} \\
& \alpha=\left[\frac{1-E\left(\widetilde{P}_{i}\right)}{E\left(\widetilde{P}_{i}\right)}\right]^{1 / 3} \times 2.301 .
\end{aligned}
$$

\section{Case Application}

Loader is one kind of machine, which integrates mechanical, electrical, and hydraulic systems. Difficulty in fault diagnosis and maintenance has greatly increased by the complexity of the system. Particularly, the normal operation of the loader is the result of the coordination among the operators, the parts of the loader, and the working environment. Due to the diversity of the loader working environment and the harmfulness of rockfall impact, only the operating condition of loaders under rockfall disasters is considered in this paper when analyzing the failure of loaders. In other words, the Bayesian network is applied to study the failure probability of loaders in rockfall disaster environment.
3.1. Structure Modeling of Bayesian Network for Loader Fault Diagnosis. The basic working principle of the loader is to amplify the power provided by the engine through the torque converter. Then, the working unit and hydraulic system are utilized to realize the loader's walking, rotating bucket, lifting arm, and other functions. Finally, the shovel and loading and unloading of materials are realized. According to $\ll$ Wheel loaders-Reliability test procedures, failure classification and evaluation 》(JB/T 12463-2015), two levels of fatal failure and major failure will cause the loader to fail directly or require immediate shutdown for maintenance. We use the above two grade principles as the evaluation criteria for loader failure. Under this judging criterion, based on a large number of accident cases and taking a wheel loader as the research object, this paper makes a comprehensive analysis of the failure causes of the loader under the rockfall disaster and establishes the Bayesian network topology structure. The specific steps are as follows:

(1) Firstly, the loader is divided into six subsystems (power unit, transmission-braking system, steering system, hydraulic system, working device, and electrical system); then Bayesian network structure is established with "abnormal fault of a subsystem" as the leaf node. The basic components and the function of each system are as follows:

(a) Power unit: The working principle of the loader power units (usually diesel engines or gasoline engines) is to provide power for other systems by fuel combustion.

(b) Transmission-brake system: The function of the transmission-brake system is to transmit the power of the power plant to the drive wheels and other operating mechanisms as required or to stop the vehicle movement. At the transmission system, failure of transmission case, torque converter, drive axle, transmission shaft, brake foot device, and hand brake have the potential to affect the failure of the wheel loader.

(c) Steering system: The function of the steering system is to change the vehicle direction according to the driver's needs. Once the system fails, the whole vehicle cannot be turned directly. At the steering system, failure of steering hydraulic pump, steering hydraulic valve, steering cylinder, and steering gear have the potential to affect the failure of wheel loader.

(d) Hydraulic system: The hydraulic system is mainly used for driving, which includes working device, hydraulic steering, and hydraulic transmission. The hydraulic system is mainly composed of hydraulic pump, working cylinder, control valve, oil tanked, and so on.

(e) Work unit: The working unit of the loader is mainly composed of bucket, connecting lever, connecting rods, rocker arm, rotary cylinder, boom cylinder, and so on.

(f) Electrical system: The loader, powered by an internal combustion engine, is composed of a 
battery, an ignition system, a control instrument, and a starting motor equipped with engines.

(2) Secondly, the Bayesian network structure was established by taking the malfunction of mechanical parts and human error as leaf nodes, respectively, which is the most direct cause of the loader's abnormal operation under rockfall disaster.

(3) Finally, a comprehensive Bayesian network structure is established with "loader abnormal operation" as the leaf node. The final Bayesian network topology of the wheeled loader is shown in Figure 3.

To express the Bayesian network structure of loaders more concisely and clearly, letters are used to represent the basic nodes ( $\mathrm{T}$ for leaf nodes, $\mathrm{M}$ for intermediate nodes, and $\mathrm{X}$ for root nodes). The code and name of $\mathrm{BN}$ nodes are shown in Table 1. It is worth noting that, to facilitate the probability analysis of the loader failure, this paper assumes that there is no interaction between the fault factors on the same layer: that is, there is no direct causal relationship between the nondescendant nodes.

It can be seen from Figure 3 that there are $36 \mathrm{BN}$ nodes in the Bayesian network of the final loader, including 1 leaf node, 8 intermediate nodes, and 27 root nodes (i.e., the most fundamental cause of loader failure). In addition, this paper only carries out the two-state analysis for each node (i.e., occurrence of 1 and no occurrence of 0 ), when the loader fault probability is analyzed.

\subsection{Parameter Modeling of Bayesian Network for Loader Fault Diagnosis}

\subsubsection{Establishing Prior Probability}

(1) Determine the Evaluation Level. Expert judgment is one of the common methods to determine prior probability under the condition of insufficient sample data. The forms of expert opinion consultation mainly include personal interviews, conference discussion, and questionnaire survey.

Delphi method is to judge the opinions and values of research objects by experts, so the selection of experts is very significant. The experts invited should be familiar with the failure mode of loading vehicles and the harmfulness of rockfall disasters and have high authority. There are several similar methods taking advantage of experts' experiences; for example, Onisawa, Zerouali, and Hamaidi [43-45] proposed choosing 3 to 5 experts to perform the judgments if there were 10-20 nodes in the network. Therefore, a total of 12 experts are invited to make a collective judgment on the occurrence probability of 27 root nodes that affect the failure of the wheel loader under rockfall disaster.

For making the prior probability of the root node more accurate, the weighted method is used to further process the evaluation results of experts. Assuming that $q$ experts are invited to evaluate $m$ events, the formula for calculating the fuzzy occurrence probability of the event $i$ is shown in equation (11).

$$
\begin{aligned}
\widetilde{P}_{i}=\sum_{k=1}^{q} \sum_{i=1}^{m} \varepsilon_{k} \otimes \widetilde{f}_{k i} & =\varepsilon_{1} \otimes \widetilde{f}_{1 i} \oplus \varepsilon_{2} \otimes \widetilde{f}_{2 i}, \ldots, \oplus \varepsilon_{q} \widetilde{f}_{2 m} \\
& =\left(P_{L i}, P_{M i}, P_{H i}\right),
\end{aligned}
$$

where $\varepsilon_{k}$ is the weight coefficient of the $k(k=1,2, \ldots, q)$ expert and $f_{k i}$ is the fuzzy value of the $k$ expert's evaluation result on the $i(i=1,2, \ldots, m)$ event.

It is worth mentioning that the weight coefficient of each expert is based on job title and the length of working experience of the experts. The basis of the specific assignment is shown in Table 2 . And the final weight value of each expert was calculated according to equation (11), which is shown in Table 3.

Because of the uncertainty of fault occurrence and experts' tendency to use natural language (i.e., the probability of occurrence is large, and the probability of occurrence is very low), the natural language should be set up to describe the possibility of occurrence of basic events in the questionnaire.

To transform the evaluation results of experts into fuzzy numbers, the probability of the wheel loader failure is divided into seven grades according to Wickens' method. In addition, to reduce the difficulty of calculation, the triangular fuzzy number $F=(l, w, u)$ is used to quantify the seven linguistic variables. The fuzzy numbers corresponding to the seven grades are very low $\{0,0,0.1\}$, low $\{0,0.1,0.3\}$, medium-low $\{0.1,0.3,0.5\}$, medium $\{0.5,0.7\}$, medium-high $\{0.5,0.7,0.9\}$, high $\{0.7,0.9,1\}$, and very high $\{0.9,1,1\}$. The membership functions are shown in Figure 4.

(2) Expert Rating. According to the set evaluation criteria, the expert scoring table is designed to target the root cause of the failure. Experts were invited to rate the occurrence probability of each node of wheeled loaders under the rockfall disaster. Due to the limit of space, Table 4 only shows part of the evaluation results.

It can be seen from Table 4 that expert 1 believes that the failure possibility of the crank connecting rod mechanism of the wheel loader (i.e., X1) is "high" in the case of rockfall disaster, which is represented by triangular fuzzy numbers $\{0.7,0.9,1.0\}$.

According to equation (11), the comprehensive fuzzy failure rate of each basic event is calculated, as shown in Table 5.

(3) Defuzzification. According to equations (8), (9), and (10), the prior probability of the root node is calculated as shown in Table 5 .

\subsubsection{Establishing a Conditional Probability Table.} Conditional probability table (CPT) represents the logical relationship among nodes in a Bayesian network, which can be obtained by combining expert judgment with actual empirical data. In the fault diagnosis of loaders, only the corresponding CPT of each fault position can be adjusted for analysis and judgment. The dependence degree of nodes in 


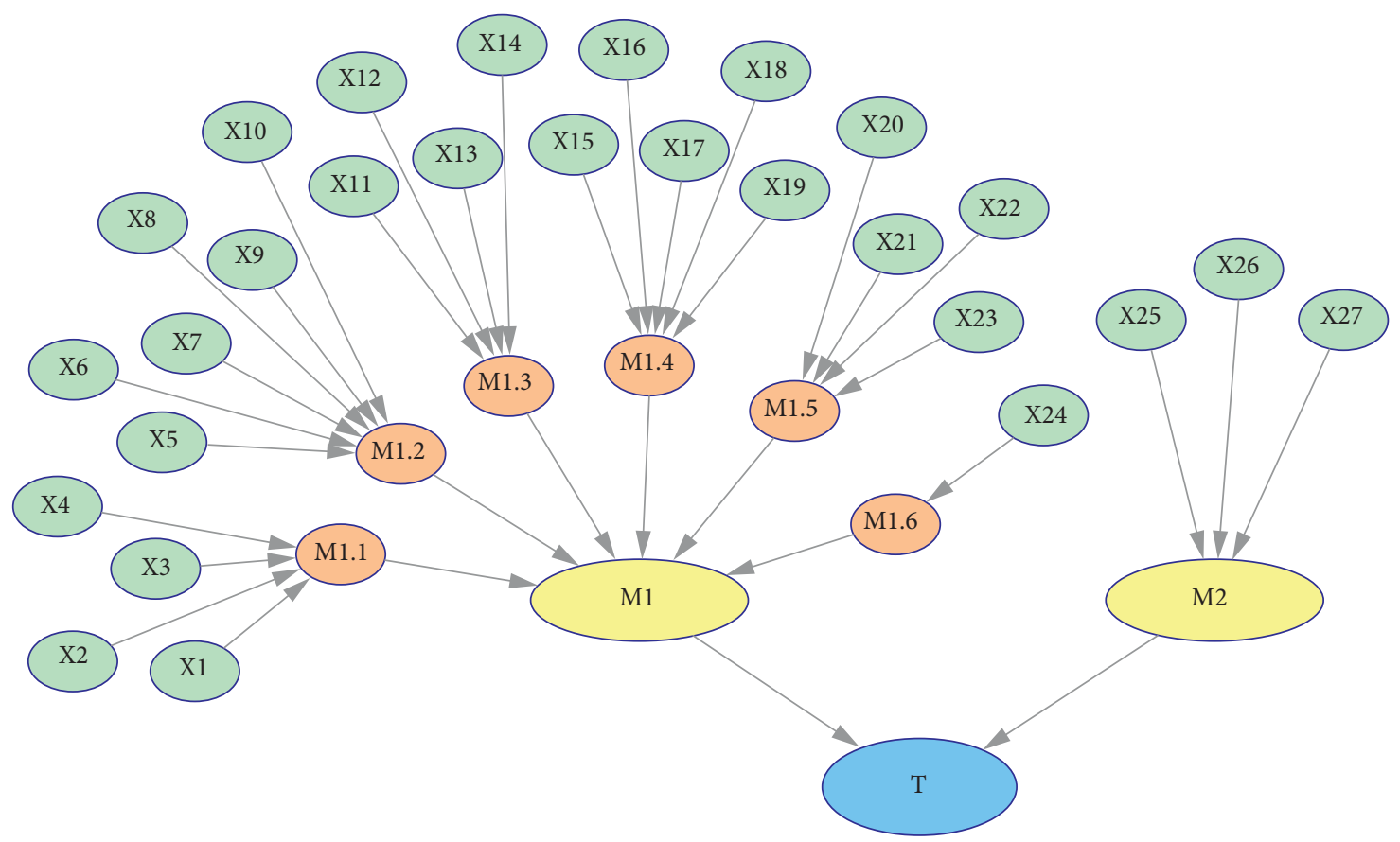

FIGURE 3: The topological structure of fault Bayesian network for wheel loader.

Bayesian networks is described by conditional probability, so it is necessary to determine all conditional probabilities between each node and its dependent nodes. There are three main methods to determine the conditional probability of each node:

(i) Identified by the researcher through a long period of observation and summary.

(ii) Determined by authoritative experts in the field.

(iii) Determined by mapping algorithms.

In practical applications, mapping the conventional fault tree (FT) to Bayesian network (BN), a convenient and effective method commonly, is widely used to apply the BN method to practical engineering problems $[15,46,47]$. This mapping algorithm not only makes up for the defect that the fault tree cannot dynamically display the dependencies between sequences but also combines the above methods and reduces the difficulty of generating CPT by Bayesian network to a great extent [48]. This paper introduced the simplified procedure of mapping FT to $\mathrm{BN}$ combined with the expert experience to determine the logic relation of each node in a Bayesian network. Details can be seen in Bobbio et al. [49].

The combination of graph mapping and numerical mapping is used to transform the fault tree into a Bayesian network and to obtain the degree of dependency among nodes. The specific transformation principle is shown in Figure 5. The upper part shows the transformation principle of graph mapping, while the lower part represents the transformation principle of numerical mapping. In this paper, the conditional probability table is obtained according to the fault tree and graph mapping principle, and the conditional probability value of each fault node of the wheel loader is obtained according to the numerical mapping of the logic gate [48].

In this paper, based on the conditional probability values of each node obtained by fault tree analysis, the corresponding conditional probability table was obtained according to the logical relationship of each node and the principle of graph mapping transformation, as shown in Figure 6. Due to the space, only the CPT corresponding to M2 (human error) was analyzed.

According to Figure 6, the failure state of each node in CPT can be represented by the variable symbols, and the logical dependence degree of each node can be represented by the conditional probability value. In this paper, $0-1$ variable is adopted to express whether the failure occurs $(0$ means no; 1 means occurrence). For example, $P\left(M_{2}=1 \mid X_{25}=0, X_{26}=1, X_{27}=1\right)=1$ means that, under the condition of node states 0 and 1 , the probability of intermediate node state 1 is equal to 1 .

3.3. Calculation of Bayesian Network for Loader Fault Diagnosis. According to the previous section, since the established Bayesian network model algorithm of the wheel loader is complex and cumbersome, the fault probability is solved with the help of GeNIe2.3 software to facilitate the analysis of the problem.

3.3.1. The Implementation Process of GeNIe. GeNIe, a flexible tool software easy to use, is developed by the Decision System Laboratory of University of Pittsburgh, which can process image models and construct the graphic decision theory models [50]. It not only supports the structure learning, parameter learning, and probability reasoning of the Bayesian network model but also has a visual interface, 
TABLE 1: The codes and names of BN nodes.

\begin{tabular}{|c|c|}
\hline Codename & Event \\
\hline $\mathrm{T}$ & Abnormal operation of the loader \\
\hline $\mathrm{M}_{1}$ & Malfunction of mechanical parts \\
\hline $\mathrm{M}_{2}$ & Error of human \\
\hline $\mathrm{M}_{1.1}^{2}$ & Failure of power unit \\
\hline $\mathrm{M}_{1.2}$ & Failure of transmission-brake system \\
\hline $\mathrm{M}_{1.3}$ & Failure of steering system \\
\hline $\mathrm{M}_{1.4}$ & Failure of hydraulic system \\
\hline $\mathrm{M}_{1.5}$ & Failure of working device \\
\hline $\mathrm{M}_{1.6}$ & Failure of electrical system \\
\hline $\mathrm{X}_{1}$ & Failure of crank connecting rod \\
\hline $\mathrm{X}_{2}$ & Failure of valve train \\
\hline $\mathrm{X}_{3}$ & Failure of fuel system \\
\hline $\mathrm{X}_{4}$ & Failure of cooling system \\
\hline $\mathrm{X}_{5}$ & Failure of transmission case \\
\hline $\mathrm{X}_{6}$ & Failure of torque converter \\
\hline $\mathrm{X}_{7}$ & Failure of drive axle \\
\hline $\mathrm{X}_{8}$ & Failure of transmission shaft \\
\hline $\mathrm{X}_{9}$ & Failure of brake foot device \\
\hline $\mathrm{X}_{10}$ & Failure of hand brake \\
\hline $\mathrm{X}_{11}$ & Failure of steering hydraulic pump \\
\hline $\mathrm{X}_{12}$ & Failure of steering hydraulic valve \\
\hline $\mathrm{X}_{13}$ & Failure of steering cylinder \\
\hline $\mathrm{X}_{14}$ & Failure of steering gear \\
\hline $\mathrm{X}_{15}$ & Failure of hydraulic pump \\
\hline $\mathrm{X}_{16}$ & Failure of hydraulic valve \\
\hline $\mathrm{X}_{17}$ & Failure of hydraulic cylinder \\
\hline $\mathrm{X}_{18}$ & Burst of oil pipe \\
\hline $\mathrm{X}_{19}$ & Failure of motor \\
\hline $\mathrm{X}_{20}$ & Cracks in working device \\
\hline $\mathrm{X}_{21}$ & Failure of driver protection structure \\
\hline $\mathrm{X}_{22}$ & Frame broken \\
\hline $\mathrm{X}_{23}$ & Cracks on the frame \\
\hline $\mathrm{X}_{24}$ & The engine cannot be started due to circuit problems \\
\hline $\mathrm{X}_{25}$ & Lack energy of the driver \\
\hline $\mathrm{X}_{26}$ & Poor psychological quality of the driver \\
\hline $\mathrm{X}_{27}$ & Command error of the commander \\
\hline
\end{tabular}

which can intuitively display the posterior probability of other nodes after a node changes.

Its specific operation process is as follows [50]:

(1) Manually set all nodes (random variables) in the topological structure of the Bayesian network by using the icon of a yellow oval from the toolbar.

(2) Connect the nodes using the arrow icon from the toolbar to define probabilistic dependence between several pairs of nodes. The specific process is to use the Node $\longrightarrow$ View as $\longrightarrow$ Bar Chart option.
TABLE 2: The expert weight assignment principle.

\begin{tabular}{lcc}
\hline Evaluation items & Grading principle & Weight coefficient \\
\hline \multirow{4}{*}{ Job title } & Professor & 5 \\
& Associate professor & 4 \\
& Associate researcher & 4 \\
& Lecturer & 2 \\
& None & 1 \\
\hline & $\geq 30$ & 5 \\
Seniority (years) & $\geq 20$ and $<30$ & 4 \\
& $>5$ and $<20$ & 3 \\
& $\leq 5$ & 2 \\
& & 1 \\
\hline
\end{tabular}

(3) Specify characteristics of a node by double-click on the particular node. In the General tab, the name and identifier of the node can be defined. In the Definition tab, one can specify the conditional probability distribution at this node. The prior probability distribution can be revealed by using the thunder icon or the option Network $\longrightarrow$ Update Immediately. Once evidence is obtained, clicking on the corresponding state of a node recalculates the posterior probabilities. The result should look similar to Figure 7.

3.3.2. Examples of Bayesian Network Reasoning. In order to help readers to understand the reasoning calculation process of the Bayesian network in the GeNIe system, this paper enumerates a modeling sample model according to the Bayesian network model of the wheel loader.

The failure of any node of Factor R1 and Factor R2 leads to the occurrence of Event L, where the probability of normal of R1 is 75\% and the probability of normal of R2 is $68 \%$. Establish the fault tree according to the above relationship. According to the conversion method in Section 3.2.2, the model is then transformed into a Bayesian network model, and the conditional probability of Factor L under the action of Factor R1 and Factor R2 is obtained. The results are shown in Figure 8 and Table 6.

The probability of the failure of the leaf node is calculated according to equation (5), and the calculation process is shown in the following formula. According to the steps in Section 3.3.1, the prior probability and conditional probability are input into the software, and the result is shown in Figure 9, which is consistent with the manual calculation result:

$$
\begin{aligned}
P(L=F)= & P(R 1=N) \times P(L=F \mid R 1=N, R 2=N) \times P(R 2=N) \\
& +P(R 1=N) \times P(L=F \mid R 1=N, R 2=F) \times P(R 2=F) \\
& +P(R 1=F) \times P(L=F \mid R 1=F, R 2=N) \times P(R 2=N) \\
& +P(R 1=F) \times P(L=F \mid R 1=F, R 2=F) \times P(R 2=F) \\
= & 0.75 \times 0 \times 0.68+0.75 \times 1 \times 0.32+0.25 \times 1 \times 0.68+0.25 \times 1 \times 0.32=0.49 .
\end{aligned}
$$


TABle 3: The composition of experts and their weight coefficients.

\begin{tabular}{|c|c|c|c|c|}
\hline Expert label & Job title & Seniority (years) & Weight score & Weight coefficient \\
\hline Expert 1 & Professor & 35 & 10 & 0.13 \\
\hline Expert 2 & Senior engineer & 16 & 8 & 0.11 \\
\hline Expert 3 & None & 7 & 3 & 0.04 \\
\hline Expert 4 & Senior engineer & 17 & 8 & 0.11 \\
\hline Expert 5 & Associate professor & 30 & 9 & 0.12 \\
\hline Expert 6 & Senior engineer & 23 & 9 & 0.12 \\
\hline Expert 7 & Lecturer & 4 & 3 & 0.04 \\
\hline Expert 8 & Senior engineer & 3 & 6 & 0.08 \\
\hline Expert 9 & None & 1 & 2 & 0.03 \\
\hline Expert 10 & Associate researcher & 4 & 5 & 0.06 \\
\hline Expert 11 & None & 15 & 4 & 0.04 \\
\hline Expert 12 & Senior engineer & 20 & 9 & 0.12 \\
\hline
\end{tabular}

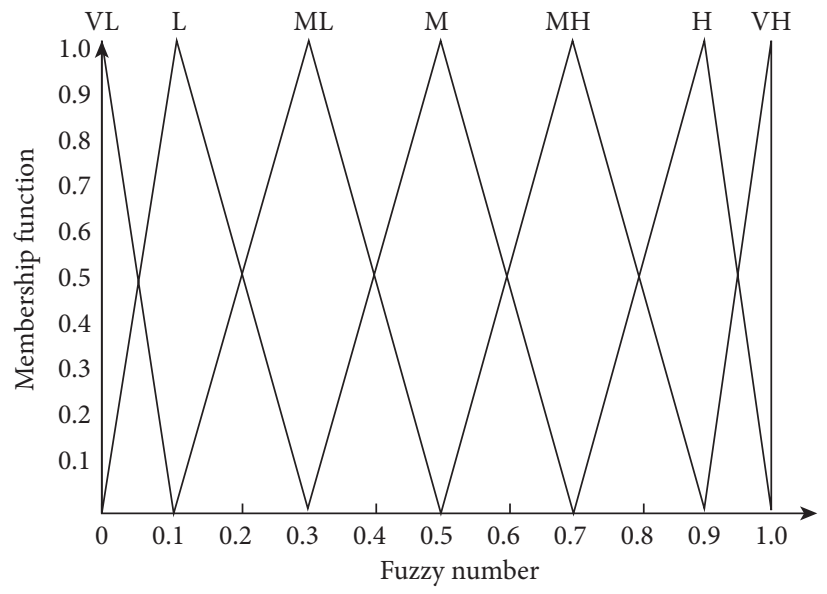

Figure 4: Membership functions.

The fault diagnosis of the Bayesian network is to observe the leaf nodes and get the fault probability of each node. The failure state of Node L is set to $100 \%$, and the fault probability of Node R1 and Node R2 can be calculated according to the Bayesian method (i.e., equation (6)). This realizes
Bayesian reverse reasoning. The reasoning result is that when the L event fails, the probability of Factor R1 failure is $51 \%$, and the probability of Factor R2 failure is $65 \%$. The calculation method is shown in the following formula:

$$
\begin{aligned}
P(R 1 & =F \mid L=F) \\
& =\frac{[P(R 1=F) \times P(L=F \mid R 1=F, R 2=N) \times P(R 2=N)+P(R 1=F) \times P(L=F \mid R 1=F, R 2=F) \times P(R 2=F)]}{P(L=F)} \\
& =\frac{(0.25 \times 0.68+0.25 \times 0.32)}{0.49}=0.51 .
\end{aligned}
$$

The results of the GeNIe2.3 calculation are shown in Figure 10, which is consistent with the manual calculation result.

\section{Results and Discussion}

4.1. Analysis of Failure Probability of Wheel Loader. For a predictive study of the failure of wheel loader investigated in detail, the Bayesian network model of wheel loader under rockfall disaster has been established, and the prior distribution of each node and CPT are determined based on expert experience and FT model. The probability of failure of the wheel loader under the condition of rockfall is obtained through the forward reasoning of the Bayesian network [27]. That is, as described in Section 2.1, causal reasoning is carried out according to the prior probability distribution of the given root node. The specific algorithm is shown in Section 3.3, and the calculation results are shown in Figure 11. 
TABLE 4: The scoring results of experts (partial).

\begin{tabular}{|c|c|c|c|c|c|}
\hline \multirow{2}{*}{ Expert label } & \multicolumn{5}{|c|}{ Evaluation object } \\
\hline & $\mathrm{X}_{1}$ & $\mathrm{X}_{2}$ & $\mathrm{X}_{3}$ & $\mathrm{X}_{4}$ & $\mathrm{X}_{5}$ \\
\hline Expert 1 & High & High & High & Medium-high & Medium-high \\
\hline Expert 2 & Medium-high & Medium-low & Medium-high & Medium-high & Medium \\
\hline Expert 3 & Low & Low & Low & Low & Low \\
\hline Expert 4 & High & Medium-high & Medium & Medium-low & High \\
\hline Expert 5 & Low & Low & Low & Low & Medium-low \\
\hline Expert 6 & Medium-high & Very high & Very high & Very high & Medium \\
\hline Expert 7 & Medium-high & High & Medium-low & Low & Medium-high \\
\hline Expert 8 & Low & Low & Low & Low & Low \\
\hline Expert 9 & Medium-low & Medium-low & Medium-low & Medium-low & Medium-low \\
\hline Expert 10 & Low & Low & Medium-low & Low & Low \\
\hline Expert 11 & Medium & Medium-low & Medium & Medium-high & Medium-low \\
\hline Expert 12 & Medium & Medium-high & Medium-low & Medium-low & Medium \\
\hline
\end{tabular}

The following conclusions can be drawn from Figure 11:

(i) The probability of failure of the wheel loader under rockfall disaster is 0.1311

(ii) The probability of failure of malfunction of mechanical parts is 0.1153

(iii) The probability of failure of human is 0.0179

\subsection{Fault Diagnosis and Analysis of the Wheel Loader}

4.2.1. Posterior Probability Calculation. When the leaf node (failure of the wheel loader) state 0 is set to $100 \%$, the probability of failure of each intermediate node and root node is determined by Bayesian network estimation, which is a posteriori reasoning. Based on Bayesian posterior reasoning, the weak links of the system can be identified and the importance of each component can be determined. In this paper, to diagnose the key cause of the failure of the wheel loader under the rockfall disaster, the posterior probability of each node is obtained by observing the "abnormal wheel loader" of the blade node.

According to Section 3.2, this paper has determined the prior distribution of each node and CPT based on expert experience and FT model, so the posterior probability can be obtained based on Bayesian method diagnosis and reasoning, of which the specific algorithm is shown in Section 3.3. In the case of known network topology, it assumes that the unknown parameters remain unchanged and finds out the possible values of all parameters. The software GeNIe is used to solve the problem, and the calculation results are shown in Figure 12.

4.2.2. Maximum Causal Chain Analysis. There are two advantages to introducing a Bayesian network into fault cause analysis: one is that fault cause factors can be represented by node variables; the other is that the state information of node variables can be used to represent the uncertain attributes of fault causes. The conditional probability between nodes can well represent the relationship between causative factors, which can reproduce the formation process of the accident in time and space.
(1) Bottom-Up Analysis. According to Figure 12, the failure of mechanical components is the main cause of loader failure under rockfall disaster. The failure probability of loaders due to mechanical component failure is as high as $88 \%$, while the probability of human error is $6 \%$.

The influence degree of six subsystems $\mathrm{M}_{1.1}-\mathrm{M}_{1.6}$ on loader accidents can be obtained through further analysis of the posterior probability in Figure 12, as shown in Figure 13.

It can be seen from Figure 13 that $\mathrm{M}_{1.2}$ (failure of transmission-brake) has the greatest impact on the loader among the six subsystems with the probability of 0.22 . Furthermore, the root node of the subsystem of $\mathrm{M}_{1.2}$ is analyzed. As shown in Figure 14, $\mathrm{X}_{9}$ (failure of Brake foot device) has the highest probability of failure, that is, 0.006423 .

(2) Top-Down Analysis. The other most approximate cause chain can be obtained by using 27 root nodes as starting points for analysis, as shown in Figure 15. The results show that the most likely causes of loader failure in rockfall disaster are $\mathrm{X}_{19}$ (failure of motor), $\mathrm{X}_{26}$ (poor psychological quality of the driver), $\mathrm{X}_{14}$ (failure of steering gear), $\mathrm{X}_{24}$ (the engine cannot be started due to circuit problems), and $\mathrm{X}_{9}$ (failure of Brake foot device).

The above analysis results are consistent with the actual situation after consulting a large number of accident investigation reports. Therefore, the maintenance of its mechanical parts should be strengthened before the loader is put into emergency rescue, which can improve the stability of the whole system under the rockfall disaster and improve the rescue efficiency. Moreover, strengthening the psychological training and management of operators should be playing a significant position to enhance their awareness of risk prevention and safety responsibility, to avoid unnecessary casualties caused by personal operational mistakes.

4.3. Summary. Through Bayesian network diagnostic reasoning, the two paths that are most likely to lead to the occurrence of the accident can be obtained from the comprehensive analysis of Figure 13 to 15 : 
TABLE 5: The prior probability of an elementary event.

\begin{tabular}{|c|c|c|c|}
\hline \multirow{2}{*}{ Basic event } & \multirow{2}{*}{ Comprehensive fuzzy failure rate } & \multicolumn{2}{|c|}{ Conversion } \\
\hline & & $E\left(\widetilde{P}_{i}\right)$ & $P_{i}$ \\
\hline $\mathrm{X} 1$ & $(0.354,0.524,0.7)$ & 0.5255 & 0.00597 \\
\hline $\mathrm{X} 2$ & $(0.36,0.518,0.677)$ & 0.51825 & 0.00568 \\
\hline $\mathrm{X} 3$ & $(0.324,0.488,0.651)$ & 0.48775 & 0.004582 \\
\hline $\mathrm{X} 4$ & $(0.274,0.428,0.604)$ & 0.4335 & 0.00305 \\
\hline $\mathrm{X} 5$ & $(0.286,0.468,0.657)$ & 0.46975 & 0.00402 \\
\hline $\mathrm{X} 6$ & $(0.27,0.452,0.648)$ & 0.4555 & 0.003614 \\
\hline $\mathrm{X} 7$ & $(0.334,0.516,0.689)$ & 0.51375 & 0.005506 \\
\hline $\mathrm{X} 8$ & $(0.3,0.476,0.654)$ & 0.4765 & 0.004224 \\
\hline X9 & $(0.362,0.539,0.705)$ & 0.53625 & 0.006423 \\
\hline $\mathrm{X} 10$ & $(0.316,0.5,0.677)$ & 0.49825 & 0.004939 \\
\hline $\mathrm{X} 11$ & $(0.378,0.537,0.686)$ & 0.5345 & 0.006348 \\
\hline $\mathrm{X} 12$ & $(0.344,0.502,0.674)$ & 0.5055 & 0.005198 \\
\hline $\mathrm{X} 13$ & $(0.287,0.468,0.644)$ & 0.46675 & 0.003931 \\
\hline $\mathrm{X} 14$ & $(0.408,0.578,0.728)$ & 0.573 & 0.008201 \\
\hline $\mathrm{X} 15$ & $(0.292,0.472,0.647)$ & 0.47075 & 0.004049 \\
\hline $\mathrm{X} 16$ & $(0.27,0.446,0.613)$ & 0.44375 & 0.003304 \\
\hline $\mathrm{X} 17$ & $(0.282,0.47,0.657)$ & 0.46975 & 0.00402 \\
\hline $\mathrm{X} 18$ & $(0.346,0.517,0.681)$ & 0.51525 & 0.005563 \\
\hline X19 & $(0.436,0.591,0.729)$ & 0.58675 & 0.008969 \\
\hline $\mathrm{X} 20$ & $(0.33,0.455,0.583)$ & 0.45575 & 0.003621 \\
\hline $\mathrm{X} 21$ & $(0.308,0.468,0.639)$ & 0.47075 & 0.004049 \\
\hline $\mathrm{X} 22$ & $(0.336,0.477,0.626)$ & 0.479 & 0.004302 \\
\hline $\mathrm{X} 23$ & $(0.362,0.491,0.62)$ & 0.491 & 0.00469 \\
\hline $\mathrm{X} 24$ & $(0.412,0.573,0.715)$ & 0.56825 & 0.00795 \\
\hline $\mathrm{X} 25$ & $(0.306,0.474,0.635)$ & 0.47225 & 0.004094 \\
\hline $\mathrm{X} 26$ & $(0.418,0.582,0.721)$ & 0.57575 & 0.00835 \\
\hline $\mathrm{X} 27$ & $(0.349,0.516,0.677)$ & 0.5145 & 0.005534 \\
\hline
\end{tabular}

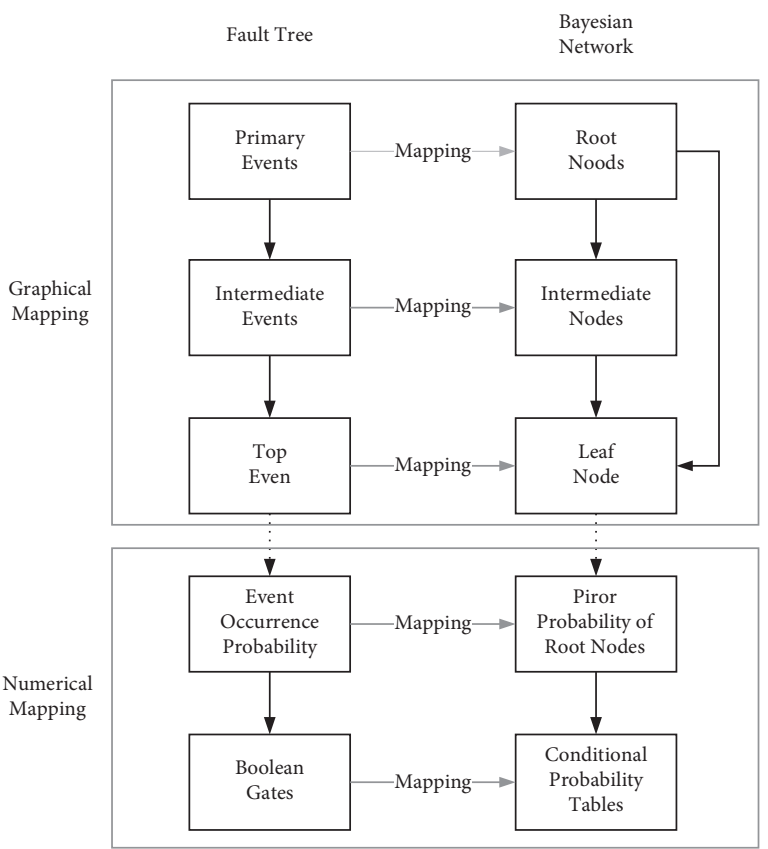

Figure 5: The conversion method from FT to BN.

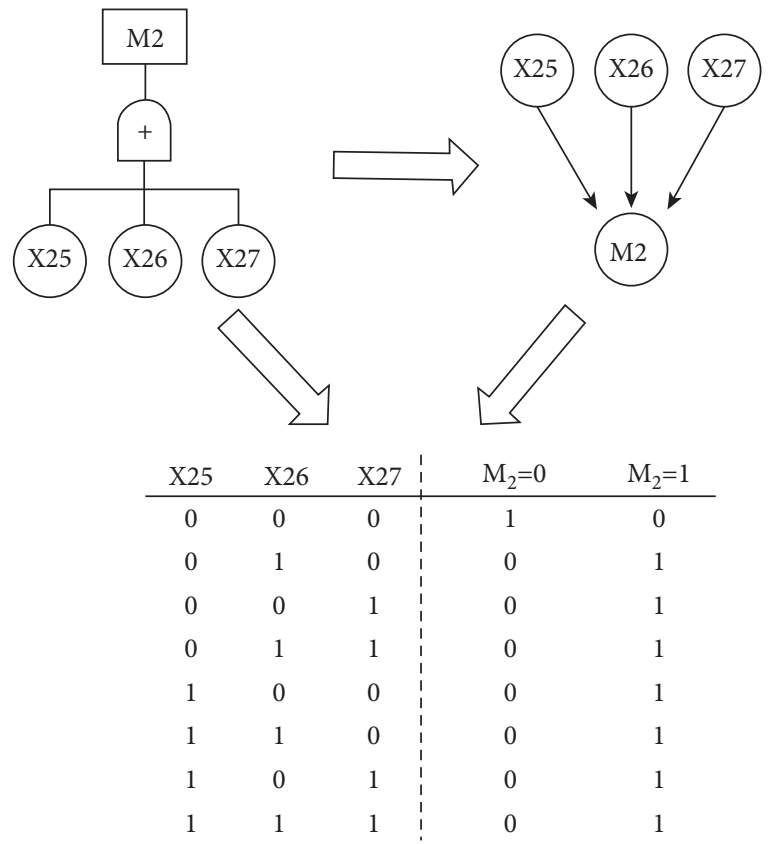

Figure 6: The conditional probability table. 


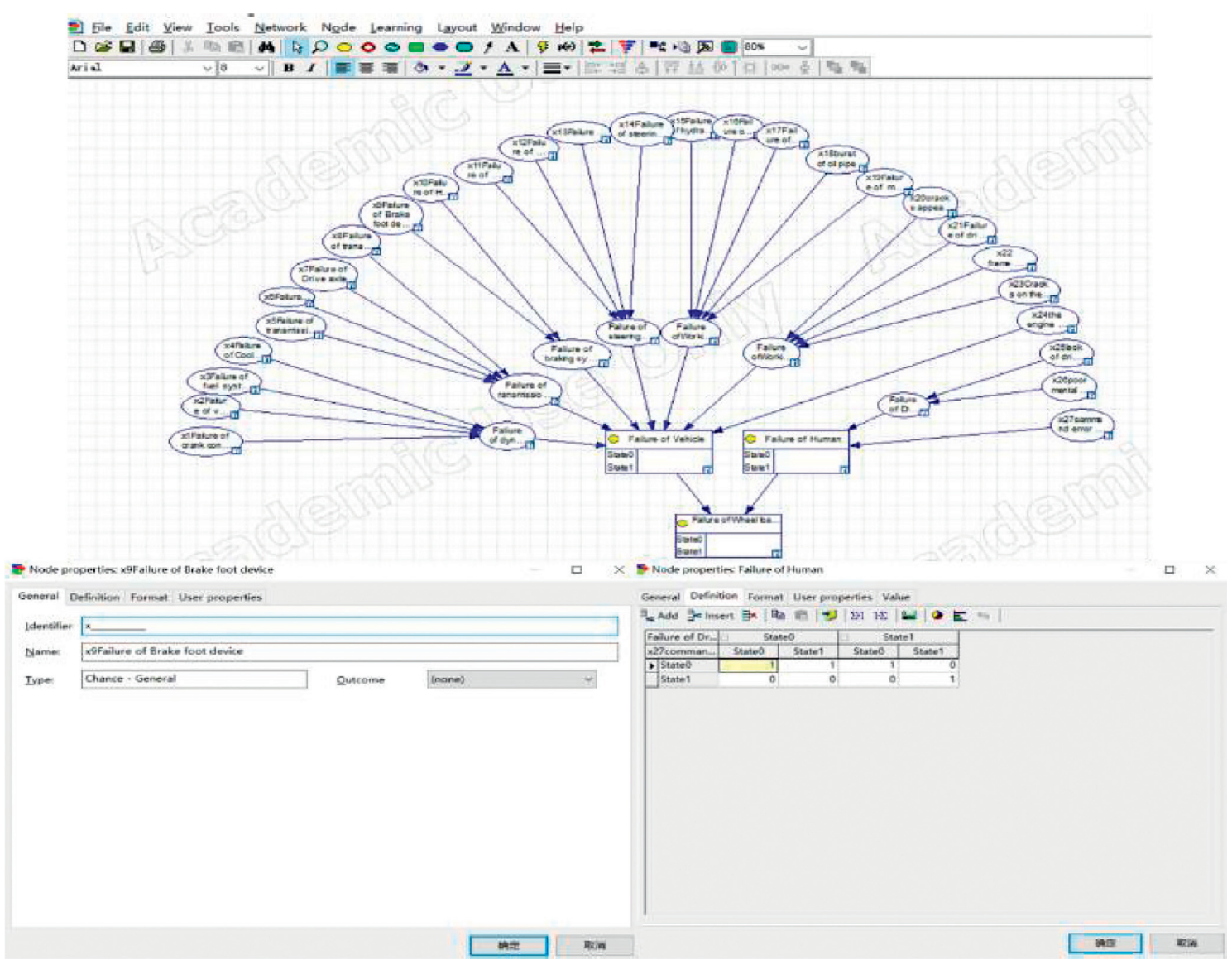

FIgURE 7: The operation interface diagram of GeNIe 2.3.

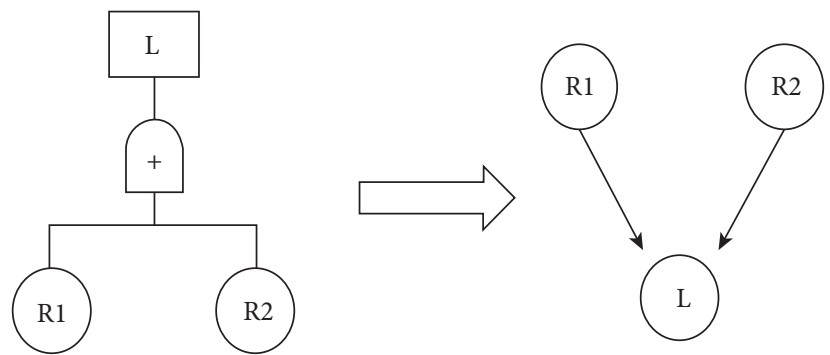

FIgURE 8: The transformation diagram of FT to BN.

TABLE 6: The CPT of $\mathrm{L}$ under the action of A and B.

\begin{tabular}{ccccccc}
\hline & R1 & & Normal & & Failure \\
& & & Failure & Normal & 0 \\
\hline R2 & & Normal & 1 & 0 & 0 & 0 \\
& & Normal & 0 & 1 & 1 & 1 \\
\hline
\end{tabular}

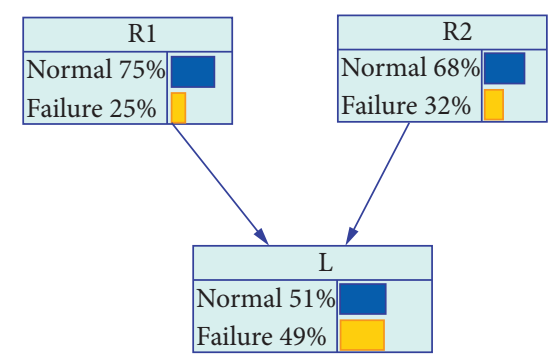

Figure 9: The failure probability of node L. 


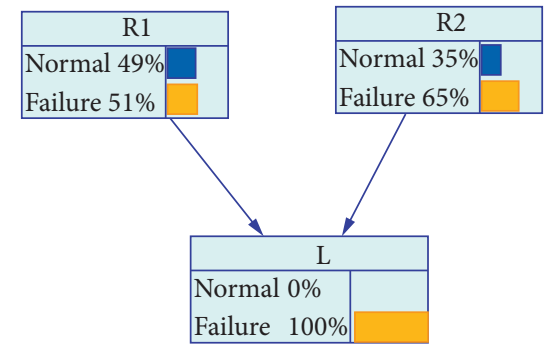

Figure 10: Posterior reasoning.

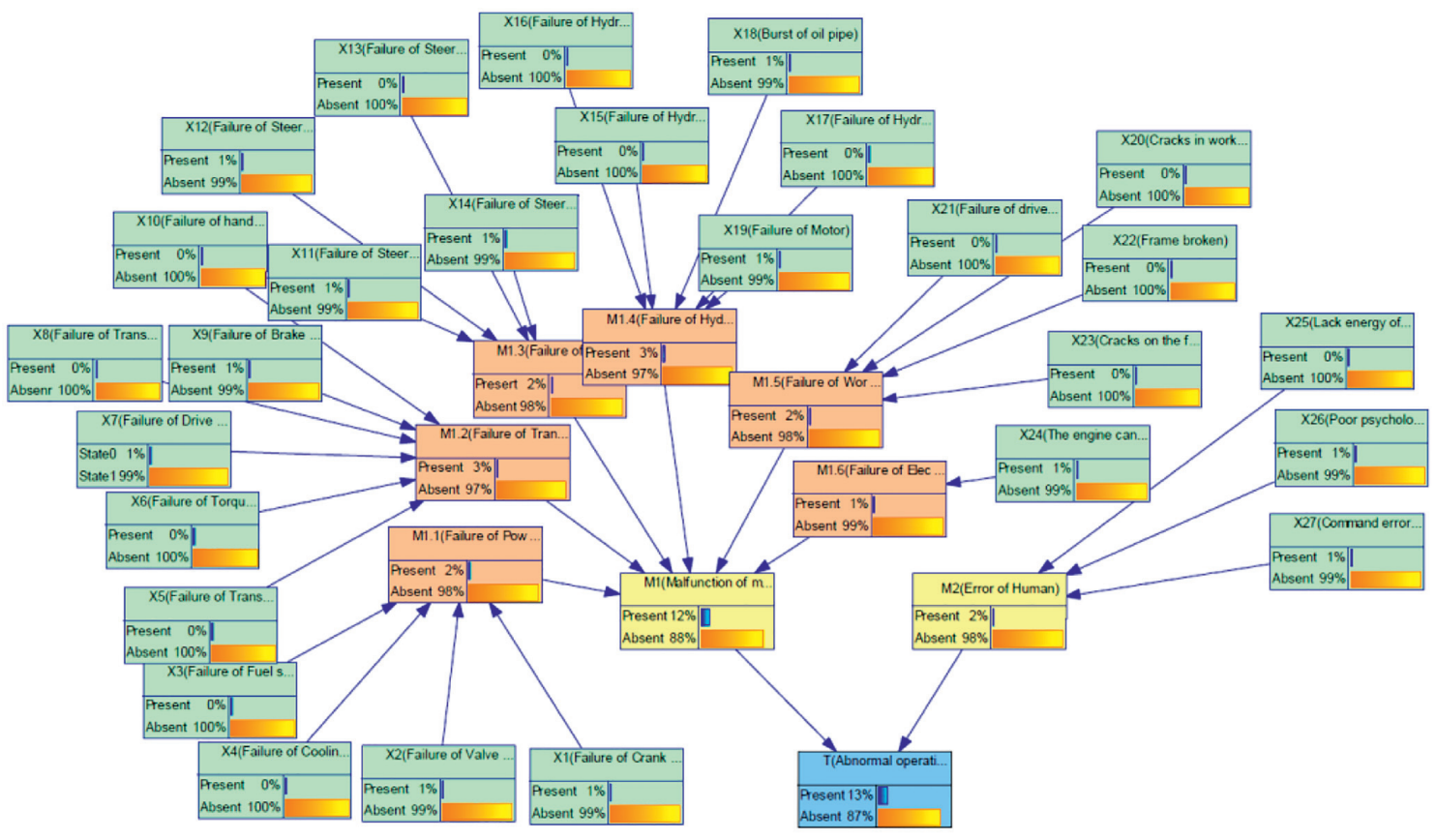

FIGURE 11: The probability of failure of the wheel loader under rockfall disaster.

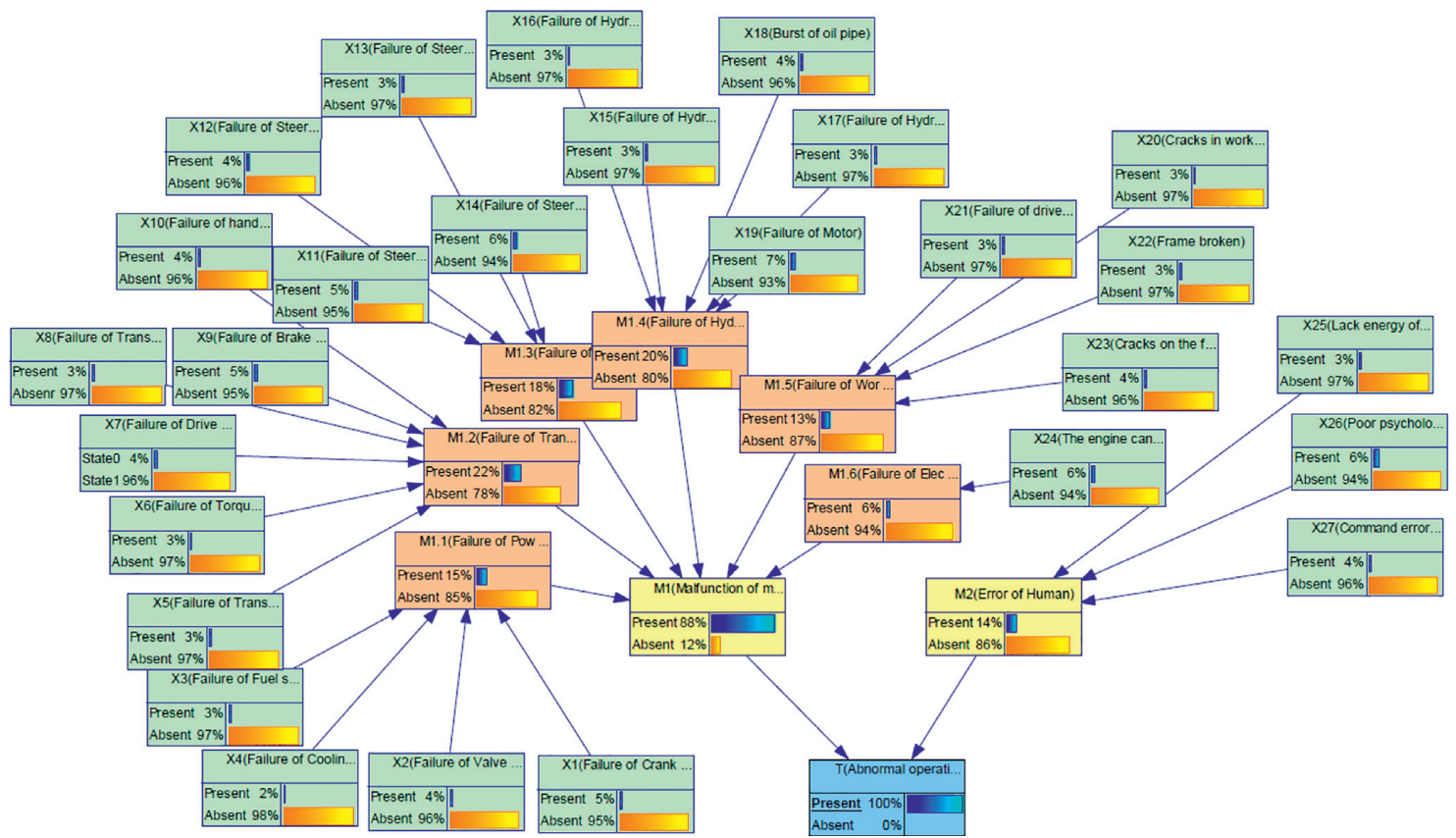

FIGURE 12: The posterior probabilities of the wheel loader under rockfall disaster. 


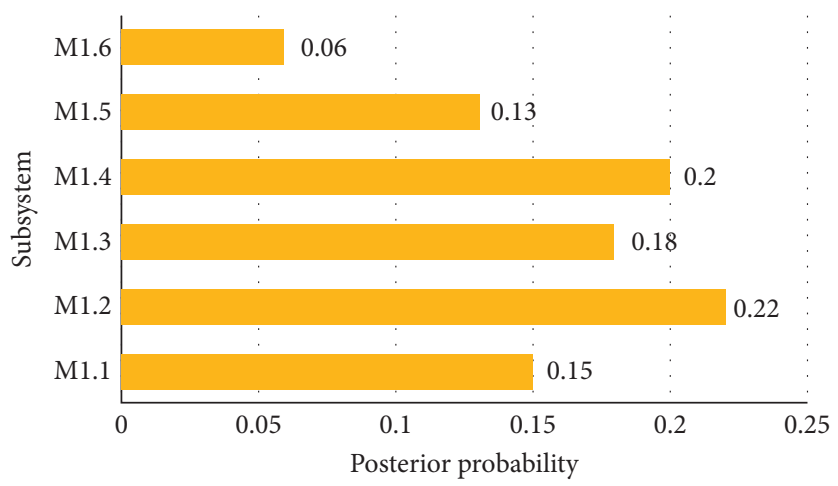

FiguRe 13: The posterior probability of $\mathrm{M}_{1.1}-\mathrm{M}_{1.6}$.

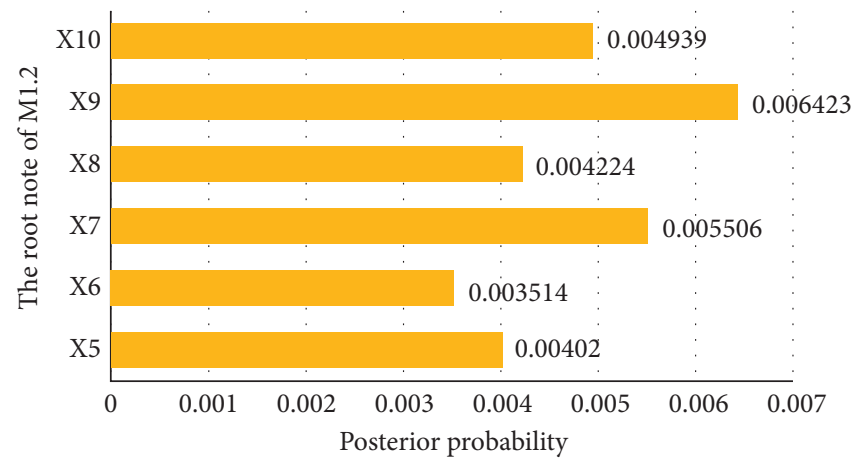

Figure 14: The posterior probability of root node of $\mathrm{M}_{1.2}$.

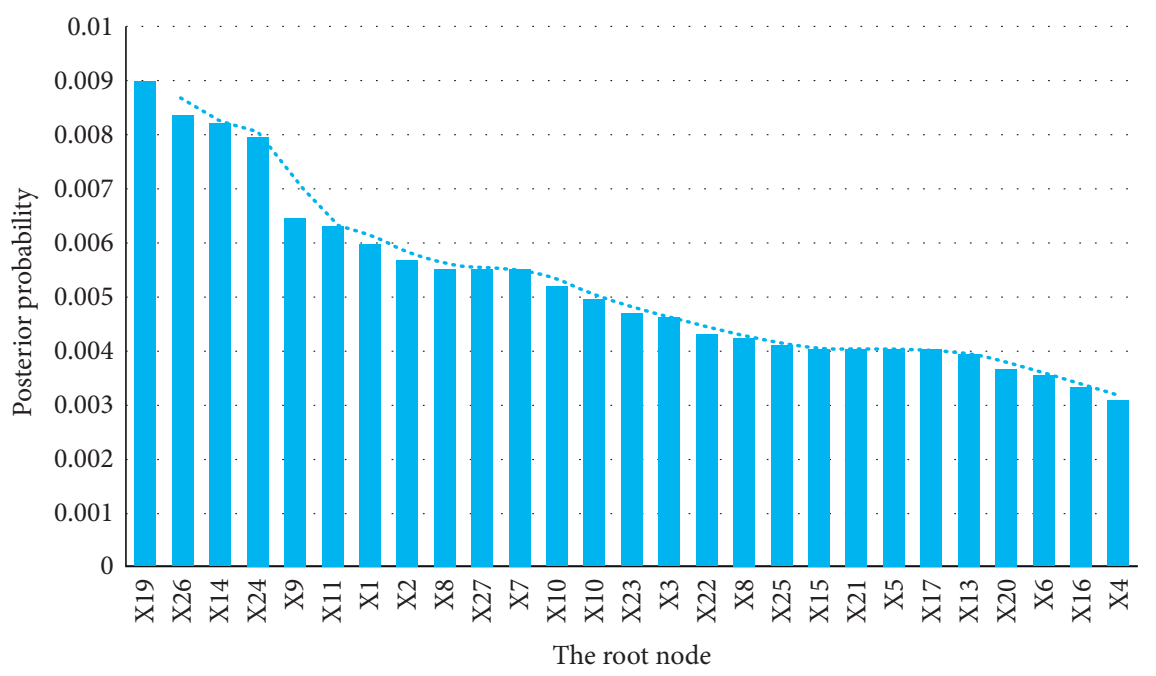

FIgURe 15: Posterior probability analysis chart of each root node.

(i) $\mathrm{X}_{9}$ (failure of brake foot device) $\longrightarrow \mathrm{M}_{1.2}$ (failure of transmission-brake) $\longrightarrow \mathrm{M}_{1}$ (malfunction of mechanical parts)

(ii) $\mathrm{X}_{19}$ (failure of motor) $\longrightarrow \mathrm{M}_{1.4}$ (failure of hydraulic system) $\longrightarrow \mathrm{M}_{1}$ (malfunction of mechanical parts)

It is reasonable to select the one with the highest posterior probability among all nodes to obtain the most approximate cause chain since the posterior probability of each node contains the relationship between the child node and the parent node.

\section{Conclusion}

In this paper, on the basis of the fault tree model of the wheel loader and expert experience, fuzzy set theory is introduced into the Bayesian network, in which fuzzy numbers are used to describe the probabilistic judgment results of each influencing factor for calculating the prior probability. In addition, the Bayesian network model was established and the simulation was carried out in the GeNIe system to obtain the cause and order of the loader failure under rockfall 
disaster. According to the calculation results, the weak links of each working system of the loader in the disaster rescue site were found, and the main conclusions were as follows:

(1) The probability of wheel loader failure under rockfall disaster is $13.11 \%$.

(2) The most likely causes of loader failure in rockfall disaster are $\mathrm{X}_{19}$ (failure of motor), $\mathrm{X}_{26}$ (poor psychological quality of the driver), $X_{14}$ (failure of steering gear), $\mathrm{X}_{24}$ (the engine cannot be started due to circuit problems), and $\mathrm{X}_{9}$ (failure of brake foot device).

(3) According to the analysis of posterior probability, two maximum cause chains of wheel loader failure are obtained: (1) $\mathrm{X}_{9}$ (failure of brake foot device) $\longrightarrow \mathrm{M}_{1.2}$ (failure of transmission-brake) $\longrightarrow \mathrm{M}_{1}$ (malfunction of mechanical parts); (2) $\mathrm{X}_{19}$ (failure of motor $) \longrightarrow M_{1.4}$ (failure of hydraulic system) $\longrightarrow M_{1}$ (malfunction of mechanical parts).

This method by fuzzy Bayesian network to analyze the failure plays a primordial role in the safety management of wheel loader under rockfalls. And, the present study provides a reference basis and valuable experience for fault identification and analysis of wheel loaders.

\section{Data Availability}

The data used to support the findings of this study are included within the article.

\section{Conflicts of Interest}

The authors declare that they have no conflicts of interest.

\section{Acknowledgments}

This work was supported by the National Natural Science Foundation of China (Grant no. 51976205) and the National Key R\&D Program of China (Grant no. 2018YFC0810600).

\section{References}

[1] S. Anne, B. Henning, C. Kristen, and M. David, "Recent rock fall activity in the Wetterstein mountains revealed by a time series of terrestrial laser scans," Mathematical Programming, vol. 119, no. 1, pp. 169-194, 2009.

[2] J. Varnes, "Slope movement types and processes," Special Report, vol. 76, pp. 11-33, 1978.

[3] Y. H. Ran, "Study on numerical simulation and protection of rock caving based on an expressway in ChongQing," Journal of Geological Hazards and Environment Preservation, vol. 030, no. 002, pp. 52-57, 2019.

[4] J. F. Wang, T. B. Li, and P. Zeng, "Research on development and distribution rules of railway collapse and rockfall disasters in Vasigou-Kangding section," High Speed Rallway Technology, vol. 11, no. 05, pp. 25-30, 2020.

[5] W. Wang, Fault Diagnosis for Loader Based on Rough Set Theory, Huazhong University of Science and Technology, Wuhan, China, 2005.
[6] S. Hang, Design and Achievement of Automatically Generated Fault Diagnosis System for Loaders, Zhejiang University, Hangzhou, China, 2018.

[7] X. Zhang, Noise Diagnosis Research on Gearbox Failure of Loader Based on ICA and SVM Algorithm, ShanDong University, Jinan, China, 2018.

[8] J. Zhao, M. Yin, and Y. B. Wei, "Fault diagnosis of loader deceleration mechanism based on transmission error method," Modular Machine Tool automatic Manufacturing Technique, no. 07, pp. 69-72, 2018.

[9] Z. Zheng, X. Shao, and D. Yu, "Fault diagnosis of a wheel loader by artificial neural networks and fuzzy logic," in Proceedings of the IEEE Conference on Robotics, Automation \& Mechatronics, IEEE, Bangkok, Thailand, June 2006.

[10] F. Xu, X. Liu, W. Chen, C. Zhou, and B. Cao, "Ontology-based method for fault diagnosis of loaders," Sensors, vol. 18, no. 3, p. 729, 2018.

[11] B. Cai, H. Lei, and X. Min, "Bayesian networks in fault diagnosis," IEEE Transactions on Industrial Informatics, vol. 13, no. 5, pp. 2227-2240, 2017.

[12] B. Cai, Y. Liu, Z. Liu, X. Tian, X. Dong, and S. Yu, "Using bayesian networks in reliability evaluation for subsea blowout preventer control system," Reliability Engineering \& System Safety, vol. 108, no. none, pp. 32-41, 2012.

[13] B. Cai, Y. Liu, Y. Ma, L. Huang, and Z. Liu, "A framework for the reliability evaluation of grid-connected photovoltaic systems in the presence of intermittent faults," Energy, vol. 93, pp. 1308-1320, 2015.

[14] H. Langseth and L. Portinale, "Bayesian networks in reliability," Reliability Engineering \& System Safety, vol. 92, no. 1, pp. 92-108, 2005.

[15] S. Barua, X. Gao, H. Pasman, and M. S. Mannan, "Bayesian network based dynamic operational risk assessment," Journal of Loss Prevention in the Process Industries, vol. 41, pp. 399410, 2016.

[16] S. H. Chen and C. A. Pollino, "Good practice in bayesian network modelling," Environmental Modelling \& Software, vol. 37, pp. 134-145, 2012.

[17] F. P. R. A. Carriger John, "Conceptual bayesian networks for contaminated site ecological risk assessment and remediation support," Journal of Environmental Management, vol. 278, 2021.

[18] F. Wittig, Learning Bayesian Networks with Hidden Variables for user Modeling, Springer Vienna, Berlin, Germany, 1999.

[19] B. Zhang, L. Z. Zhou, and C. C. Si, "Research on corruption vulnerability of university construction project based on bayesian Network," Journal of Engineering Management, vol. 34, no. 06, pp. 25-30, 2020.

[20] G. Kabir, R. Sadiq, and S. Tesfamariam, "A fuzzy bayesian belief network for safety assessment of oil and gas pipelines," Structure and Infrastructure Engineering, vol. 12, no. 8, 2016.

[21] P. Han, M. Q. Wang, and Y. F. Zhao, "Failure risk assessment of logistics UAV based on bayesian network," Journal of Safety Science and Technology, vol. 16, no. 11, pp. 178-183, 2020.

[22] M. Yazdi and S. Kabir, "A fuzzy bayesian network approach for risk analysis in process industries," Process Safety and Environmental Protection, vol. 111, pp. 507-519, 2017.

[23] G. Zhang, V. V. Thai, K. F. Yuen, H. S. Loh, and Q. Zhou, "Addressing the epistemic uncertainty in maritime accidents modelling using bayesian network with interval probabilities," Safety Science, vol. 102, pp. 211-225, 2018.

[24] S. T. Zheng, Bayesian Network-Based Reliability Analysis of Metro Train Brake System, Beijing Jiaotong University, Beijing, China, 2018. 
[25] F. V. Jensen, An Introduction to Bayesian Networks, UCL Press, London, UK, 1996.

[26] L. Portinale, D. C. Raiteri, and S. Montani, "Supporting reliability engineers in exploiting the power of dynamic bayesian networks," International Journal of Approximate Reasoning, vol. 51, no. 2, pp. 179-195, 2010.

[27] C. Lin, Reliability Analysis of Bus Braking System Based on Bayesian Network, Chang'an University, Xian, China, 2016.

[28] X. Jiang and S. Mahadevan, "Bayesian structural equation modeling method for hierarchical model validation," Reliability Engineering and System Safety, vol. 94, no. 4, 2008.

[29] Y. L. Hsu, C. H. Lee, and V. B. Kreng, "The application of fuzzy delphi method and fuzzy AHP in lubricant regenerative technology selection," Expert Systems with Applications, vol. 37, no. 1, pp. 419-425, 2010.

[30] H. Ishibuchi, K. Kwon, and H. Tanaka, "A learning algorithm of fuzzy neural networks with triangular fuzzy weights," Fuzzy Sets and Systems, vol. 71, no. 3, pp. 277-293, 1995.

[31] M. Wang and D. J. R. E. Niu, "Research on project postevaluation of wind power based on improved ANP and fuzzy comprehensive evaluation model of trapezoid subordinate function improved by interval number," Renewable Energy, vol. 132, 2019.

[32] G. Madhu, Gaussian Membership Function and Type II Fuzzy Sets Based Approach for Edge Enhancement of Malaria Parasites in Microscopic Blood Images, Springer, Berlin, China, 2018.

[33] C. Wickens, Engineering Psychology and Human Performance, Harper Collins Publishers, New York, NY, USA, 1984.

[34] A. S. Markowski and M. S. Mannan, "Fuzzy risk matrix," Journal of Hazardous Materials, vol. 159, no. 1, pp. 152-157, 2008.

[35] R. Ferdous, F. Khan, R. Sadiq, P. Amyotte, and B. Veitch, "Analyzing system safety and risks under uncertainty using a bow-tie diagram: an innovative approach," Process Safety Environmental Protection, vol. 91, no. 1-2, 2013.

[36] S. M. Lavasani, N. Ramzali, F. Sabzalipour, and E. Akyuz, "Utilisation of fuzzy fault tree analysis (FFTA) for quantified risk analysis of leakage in abandoned oil and natural-gas wells," Ocean Engineering, vol. 108, no. NOV.1, pp. 729-737, 2015.

[37] A. Mardani, A. Jusoh, and E. K. Zavadskas, "Fuzzy multiple criteria decision-making techniques and applications two decades review from 1994 to 2014," Expert Systems with Applications, vol. 42, no. 8, pp. 4126-4148, 2015.

[38] J. H. Purba, J. Lu, G. Zhang, and W. Pedrycz, "A fuzzy reliability assessment of basic events of fault trees through qualitative data processing," Fuzzy Sets and Systems, vol. 243, no. may 16, pp. 50-69, 2014.

[39] W. Pedrycz, "Why triangular membership functions," Fuzzy Sets and Systems, vol. 64, no. 1, pp. 21-30, 1994.

[40] K. Wang, Reliability Analysis of Mine Braking System Based on Bayesian Network, Taiyuan University of Science and Technology, Taiyuan, China, 2019.

[41] L. Zhang, M. J. Skibniewski, X. Wu, Y. Chen, and Q. Deng, "A probabilistic approach for safety risk analysis in metro construction," Safety Science, vol. 63, no. 3, pp. 8-17, 2014.

[42] R. R. Yager, "A procedure for ordering fuzzy subsets of the unit interval," Information Sciences, vol. 24, no. 2, pp. 143-161, 1981.

[43] T. Onisawa, "An approach to human reliability in manmachine systems using error possibility," Fuzzy Sets and Systems, vol. 27, no. 2, pp. 87-103, 1988.
[44] B. Zerouali and B. Hamaidi, "Predictive analysis for risk of fire and explosion of LNG storage tanks by fuzzy Bayesian network," Life Cycle Reliability and Safety Engineering,vol. 9, pp. 1-10, 2019.

[45] T. Hao and L. Shi, "Basic theory of fuzzy bayesian networks and its application in machinery fault diagnosis," in Proceedings of the Fourth International Conference on Fuzzy Systems and Knowledge Discovery, 2007 (FSKD 2007), IEEE, Haikou, China, August 2007.

[46] M. Yazdi and S. Kabir, "A fuzzy bayesian network approach for risk analysis in process industries," Process Safety and Environmental Protection, vol. 111, 2017.

[47] M. Haenmnen, O. Banda, and P. Kujala, "Bayesian network model of maritime safety management," Expert Systems with Applications, vol. 41, no. 17, pp. 7837-7846, 2014.

[48] N. Khakzad, F. Khan, and P. Amyotte, "Dynamic safety analysis of process systems by mapping bow-tie into Bayesian network," Process Safety Environmental Protection, vol. 91, no. 1-2, pp. 46-53, 2013.

[49] A. Bobbio, L. Portinale, M. Minichino, and E. Ciancamerla, "Improving the analysis of dependable systems by mapping fault trees into bayesian networks," Reliability Engineering \& System Safety, vol. 71, no. 3, pp. 249-260, 2001.

[50] M. J. Druzdzel, "SMILE: structural modeling, inference, and learning engine and GeNIE: a development environment for graphical decision-theoretic models," in Proceedings of the Sixteenth National Conference on Artificial Intelligence \& Eleventh Conference on Innovative Applications of Artificial Intelligence, Orlando, FL, USA, January 1999. 